Discrete Comput Geom 31:357-367 (2004)

DOI: $10.1007 / \mathrm{s} 00454-003-2880-2$

\title{
An Inequality for Circle Packings Proved by Semidefinite Programming
}

\author{
Pablo A. Parrilo ${ }^{1}$ and Ronen Peretz ${ }^{2}$ \\ ${ }^{1}$ Automatic Control Laboratory, ETH-Zentrum, \\ CH-8092 Zürich, Switzerland \\ parrilo@control.ee.ethz.ch \\ ${ }^{2}$ Department of Mathematics, Ben Gurion University of the Negev, \\ Beer-Sheva 84105, Israel \\ ronenp@math.bgu.ac.il
}

\begin{abstract}
A geometric inequality among three triangles, originating in circle packing problems, is introduced. In order to prove it, we reduce the original formulation to the nonnegativity of a polynomial in four real indeterminates. Techniques based on sum of squares decompositions, semidefinite programming and symmetry reduction are then applied to provide an easily verifiable nonnegativity certificate.
\end{abstract}

\section{Introduction}

In this paper we prove the following geometric inequality: suppose that we have three triangles, one with sides of lengths $X, Y$ and $Z$, a second with sides of lengths $U, V$ and $W$ and a third triangle with sides of lengths $(X+U),(Y+V)$ and $(Z+W)$. We denote by $\alpha$ the angle in the first triangle between the sides of lengths $X$ and $Y$. Let $\beta$ be the corresponding angle in the second triangle between $U$ and $V$ and let $\gamma$ be the corresponding angle between $(X+U)$ and $(Y+V)$ in the third triangle. Then

$$
\alpha \cdot(X+Y-Z)+\beta \cdot(U+V-W) \leq \gamma \cdot((X+U)+(Y+V)-(Z+W))
$$

The importance of this inequality stems from a new approach to circle packings problems, introduced in [10]. As we will see in Section 2, a crucial superadditivity property needed in that approach is implied by the above geometric inequality. It turns out that proving (1) it is not at all simple.

A crucial feature of our proof of this inequality is the use of recent algorithms, based on semidefinite programming (SDP), for producing representations of nonnegative 
polynomials as sums of squares [4], [9]. As is well known, questions about polynomial nonnegativity (or, more generally, about semialgebraic sets) are an essential component in many geometric problems. Efficient algorithmic approaches to deal with these kinds of issues are therefore of great practical and theoretical importance. We are convinced that the general techniques illustrated in this paper, namely sum of squares decompositions, SDP and symmetry reduction, have a lot of potential in this area and provide a sound general approach for many other problems of similar geometric nature.

As we will show, inequality (1) holds true if the polynomial

$$
\begin{aligned}
L= & \alpha^{2} \beta^{2}(\alpha-\beta)^{2}+\beta^{2}(1-\alpha \beta)\left(1+\alpha \beta-2 \alpha^{2}\right) \gamma^{2} \\
& +\alpha^{2}(1-\alpha \beta)\left(1+\alpha \beta-2 \beta^{2}\right) \delta^{2}-\alpha \beta\left(2+\alpha \beta^{3}-4 \alpha \beta+\beta \alpha^{3}\right) \gamma \delta \\
& +\beta(1-\alpha \beta)\left(2 \alpha-\beta-\alpha \beta^{2}\right) \gamma^{3} \delta-\left(\alpha^{2}+\beta^{2}+2 \alpha^{3} \beta^{3}-4 \alpha^{2} \beta^{2}\right) \gamma^{2} \delta^{2} \\
& +\alpha(1-\alpha \beta)\left(2 \beta-\alpha-\alpha^{2} \beta\right) \gamma \delta^{3}+(\alpha-\beta)^{2} \gamma^{3} \delta^{3}
\end{aligned}
$$

is nonnegative on the open unit box $0<\alpha, \beta, \gamma, \delta<1$. At this point, we encourage the reader to try to prove the nonnegativity of $L$, to get a full appreciation of the difficulty of the problem.

To prove the inequality, we make a further reduction to the unconstrained nonnegativity of a certain real polynomial of degree 20 in four real indeterminates, and attempt to express it as a sum of squares (SOS). Although unstructured instances of this size are near the limits of what can be achieved using generic SOS/SDP methods, our particular polynomial enjoys certain convenient sparsity and symmetry properties. These allow the use of new algorithms, introduced in [5], customized for polynomials with an invariant structure. Even though the computational procedures in its current form use floating point arithmetic to arrive at the result, our final solution can be easily verified in a completely independent fashion. The methods work quite nicely in the problem at hand, producing a concise representation of the polynomial $L$ as

$$
\begin{aligned}
& L=L_{1}+L_{2}+L_{3}, \\
& L_{1}=(\gamma+\delta)\left(-\alpha^{2} \beta+\alpha \beta^{2}-\alpha \delta+\beta \gamma-\beta \gamma \delta+\alpha \delta \gamma-\alpha \beta^{2} \gamma+\alpha^{2} \beta \delta\right)^{2}, \\
& L_{2}=(1-\gamma)(1-\delta)(\alpha \beta-1)^{2}(\alpha \delta-\beta \gamma)^{2}, \\
& L_{3}=(1-\gamma)(1-\delta)(\alpha-\beta)^{2}(\alpha \beta-\gamma \delta)^{2},
\end{aligned}
$$

which is an easily verifiable certificate of its nonnegativity on the unit box.

In the remainder of the paper we provide some background on the problem, and explain in detail the reduction of the original inequality to the algebraic characterization (2), as well as the sum of squares and symmetry reduction techniques employed in arriving at the final certificate (3).

\section{The Superadditivity of $f_{\bar{a}}$}

The need for our inequality originates in [10]. This last paper describes a new approach to circle packings [1], [7], [12], with the main features being the theory of Perron-Frobenius for nonnegative matrices [3], [6], [13] and fixed-point theory [8], [2]. In particular, our approach uses a converse of the contraction principle as it appears in [2]. A central object 
that is introduced in [10] is the $\bar{a}$-mapping, $f_{\bar{a}}: \mathbb{R}_{+}^{|V|} \rightarrow \mathbb{R}_{+}^{|V|}$, of a graph embedding. This is a variant of Thurston's relaxation mapping. A key property of the $\bar{a}$-mapping is its superadditivity,

$$
\forall \bar{r}, \bar{s} \in \mathbb{R}_{+}^{|V|}, \quad f_{\bar{a}}(\bar{r})+f_{\bar{a}}(\bar{s}) \leq f_{\bar{a}}(\bar{r}+\bar{s}) .
$$

As proved in [10], the superadditivity of $f_{\bar{a}}$ follows from

Theorem 1. If $a, b, c, d, R, S>0$, then

$$
\begin{aligned}
R \sin ^{-1}\left\{\sqrt{\frac{a b}{(R+a)(R+b)}}\right\}+S \sin ^{-1}\left\{\sqrt{\frac{c d}{(S+c)(S+d)}}\right\} \\
\leq(R+S) \sin ^{-1}\left\{\sqrt{\frac{(a+c)(b+d)}{(R+S+a+c)(R+S+b+d)}}\right\} .
\end{aligned}
$$

The theorem has two simple geometric interpretations:

(1) Three circles of radii $R, a$ and $b$ that are mutually tangent to one another from the outside form a Euclidean triangle. The vertices of the triangle are the centers of the circles. The sides of the triangle have the following lengths: $R+a, a+b$ and $R+b$. Similarly, three circles of radii $S, c$ and $d$ form a triangle of sides $S+c, c+d$ and $S+d$. Finally, a third such triangle is formed by three circles of radii $R+S+a+c$, $a+c+b+d$ and $R+S+b+d$. We note that the sides of the third triangle have lengths which are the sums of the corresponding sides of the first two triangles. On the other hand, the three sets of triples of circles also form three circular triangles. The vertices of these triangles are the tangency points of pairs of circles in each triple. The lemma implies that the circular sides of the third (largest) triangle are greater than or equal to the sums of the corresponding circular sides of the first two circular triangles.

(2) We consider three Euclidean triangles. One with sides of lengths $X, Y$ and $Z$, and an angle $\alpha$ between $X$ and $Y$. A second triangle with sides of lengths $U, V$ and $W$, and an angle $\beta$ between $U$ and $V$. A third triangle with sides of lengths $(X+U),(Y+V)$ and $(Z+W)$, and an angle $\gamma$ between $(X+U)$ and $(Y+V)$. Then

$$
\alpha \cdot(X+Y-Z)+\beta \cdot(U+V-W) \leq \gamma \cdot((X+U)+(Y+V)-(Z+W)) .
$$

The latter interpretation corresponds exactly to our inequality as presented in the Introduction.

\section{Reducing to Algebraic Inequalities}

We now make a reduction of the inequality of Theorem 1 . There are two ideas involved in it. The first idea is summarized in the following:

Lemma 1. Suppose that there exists a twice differentiable, surjective and strictly 
increasing function $f: I \rightarrow[0,1]$ which satisfies the following two conditions:

(i)

$$
f^{\prime \prime}\left(1-f^{2}\right)+f \cdot\left(f^{\prime}\right)^{2} \leq 0 \quad \text { on } I .
$$

$$
\begin{gathered}
\left(\frac{R}{R+S}\right) f^{-1}\left(\sqrt{\frac{a b}{(R+a)(R+b)}}\right)+\left(\frac{S}{R+S}\right) f^{-1}\left(\sqrt{\frac{c d}{(S+c)(S+d)}}\right) \\
\leq f^{-1}\left(\sqrt{\frac{(a+c)(b+d)}{(R+S+a+c)(R+S+b+d)}}\right),
\end{gathered}
$$

for all $a, b, c, d, R, S>0$. Then the inequality of Theorem 1 holds true.

Proof. Consider the function $y=\sin ^{-1} f(x)$ for $x \in I$. Then

$$
\begin{aligned}
\frac{d y}{d x} & =\frac{f^{\prime}}{\sqrt{1-f^{2}}}, \\
\frac{d^{2} y}{d x^{2}} & =\frac{f^{\prime \prime}\left(1-f^{2}\right)+f \cdot\left(f^{\prime}\right)^{2}}{\left(1-f^{2}\right)^{3 / 2}} .
\end{aligned}
$$

By condition (i) we get $d^{2} y / d x^{2} \leq 0$ on $I$ and hence $y$ is concave in $I$. So for any $x, z \in I$ and for any $0 \leq t \leq 1$, we have

$$
t \sin ^{-1} f(x)+(1-t) \sin ^{-1} f(z) \leq \sin ^{-1} f(t x+(1-t) z) .
$$

We make the following choice:

$$
x=f^{-1}\left(\sqrt{\frac{a b}{(R+a)(R+b)}}\right), \quad z=f^{-1}\left(\sqrt{\frac{c d}{(S+c)(S+d)}}\right), \quad t=\left(\frac{R}{R+S}\right) .
$$

Then, by inequality (4), we get

$$
\begin{gathered}
\left(\frac{R}{R+S}\right) \sin ^{-1}\left(\sqrt{\frac{a b}{(R+a)(R+b)}}\right)+\left(\frac{S}{R+S}\right) \sin ^{-1}\left(\sqrt{\frac{c d}{(S+c)(S+d)}}\right) \\
\leq \sin ^{-1} f(t x+(1-t) z) .
\end{gathered}
$$

By condition (ii) we have

$$
t x+(1-t) z \leq f^{-1}\left(\sqrt{\frac{(a+c)(b+d)}{(R+S+a+c)(R+S+b+d)}}\right)
$$

and since $f$ is increasing and $\sin ^{-1}$ is also increasing, we get

$$
\sin ^{-1} f(t x+(1-t) y) \leq \sin ^{-1}\left(\sqrt{\frac{(a+c)(b+d)}{(R+S+a+c)(R+S+b+d)}}\right) .
$$

Theorem 1 follows by inequalities (5) and (6). 


\section{Special Cases}

Case (I): $f(x)=\sin x, I=[0, \pi / 2] . \quad$ Then in this case we have

$$
f^{\prime \prime}\left(1-f^{2}\right)+f \cdot\left(f^{\prime}\right)^{2}=-\sin x \cos ^{2} x+\sin x \cos ^{2} x \equiv 0
$$

and condition (i) of the lemma is satisfied. Condition (ii) is the inequality of Theorem 1 and so the theorem is trivially correct in this case.

Case (II): $f(x)=1-1 / x, I=[1, \infty] . \quad$ Then in this case we have

$$
\begin{aligned}
f^{\prime \prime}\left(1-f^{2}\right)+f \cdot\left(f^{\prime}\right)^{2} & =-\frac{2}{x^{3}}\left(1-\left(1-\frac{1}{x}\right)^{2}\right)+\left(1-\frac{1}{x}\right) \cdot \frac{1}{x^{4}} \\
& =\frac{1}{x^{5}}-\frac{3}{x^{4}}<0,
\end{aligned}
$$

for $x \geq 1$. So condition (i) is satisfied. Condition (ii) and the conclusion of the theorem prove the following:

Lemma 2. If for every $a, b, c, d, R, S>0$ the following inequality is true,

$$
\begin{aligned}
\left(\frac{R}{R+S}\right)( & \left.\frac{1}{1-\sqrt{(a b) /[(R+a)(R+b)]}}\right) \\
& +\left(\frac{S}{R+S}\right)\left(\frac{1}{1-\sqrt{(c d) /[(S+c)(S+d)]}}\right) \\
\leq & \frac{1}{1-\sqrt{[(a+c)(b+d)] /[(R+S+a+c)(R+S+b+d)]}},
\end{aligned}
$$

then the inequality of Theorem 1 is true.

The second idea in this approach (after that of Lemma 1) is an elementary trick to eliminate the square root functions in Lemma 2. We denote

$$
\alpha=\sqrt{\frac{a}{R+a}}, \quad \beta=\sqrt{\frac{b}{R+b}}, \quad \gamma=\sqrt{\frac{c}{S+c}}, \quad \delta=\sqrt{\frac{d}{S+d}} .
$$

Then $0 \leq \alpha, \beta, \gamma, \delta \leq 1$. Also $\alpha, \beta$ are independent except for $\alpha=1$ iff $\beta=1$. That happens only if $R=0 . \gamma, \delta$ are independent except for $\gamma=1$ iff $\delta=1$. That happens only if $S=0$. For the inverse transformations we have

$$
a=\left(\frac{\alpha^{2}}{1-\alpha^{2}}\right) R, \quad b=\left(\frac{\beta^{2}}{1-\beta^{2}}\right) R, \quad c=\left(\frac{\gamma^{2}}{1-\gamma^{2}}\right) S, \quad d=\left(\frac{\delta^{2}}{1-\delta^{2}}\right) S .
$$

With these notations, the left-hand side of the inequality in Lemma 2 is

$$
\left(\frac{R}{R+S}\right)\left(\frac{1}{1-\alpha \beta}\right)+\left(\frac{S}{R+S}\right)\left(\frac{1}{1-\gamma \delta}\right)=\frac{R(1-\gamma \delta)+S(1-\alpha \beta)}{(R+S)(1-\alpha \beta)(1-\gamma \delta)}
$$


As for the right-hand side, we have

$$
\begin{gathered}
I_{1}=\sqrt{\frac{a+c}{R+S+a+c}}=\sqrt{\frac{\alpha^{2}\left(1-\gamma^{2}\right) R+\gamma^{2}\left(1-\alpha^{2}\right) S}{\left(1-\gamma^{2}\right) R+\left(1-\alpha^{2}\right) S}}, \\
I_{2}=\sqrt{\frac{b+d}{R+S+b+d}}=\sqrt{\frac{\beta^{2}\left(1-\delta^{2}\right) R+\delta^{2}\left(1-\beta^{2}\right) S}{\left(1-\delta^{2}\right) R+\left(1-\beta^{2}\right) S}} .
\end{gathered}
$$

Plugging these into the inequality of Lemma 2 we get

$$
\frac{R(1-\gamma \delta)+S(1-\alpha \beta)}{(R+S)(1-\alpha \beta)(1-\gamma \delta)} \leq \frac{1}{1-I_{1} I_{2}} .
$$

Hence,

$$
I_{1} I_{2} \geq \frac{R \alpha \beta(1-\gamma \delta)+S \gamma \delta(1-\alpha \beta)}{R(1-\gamma \delta)+S(1-\alpha \beta)} .
$$

On squaring both sides we conclude that in order to prove Theorem 1 , it suffices to prove the following:

Lemma 3. If $R, S>0$ and $0<\alpha, \beta, \gamma, \delta<1$, then

$$
\begin{gathered}
\left(\frac{\alpha^{2}\left(1-\gamma^{2}\right) R+\gamma^{2}\left(1-\alpha^{2}\right) S}{\left(1-\gamma^{2}\right) R+\left(1-\alpha^{2}\right) S}\right)\left(\frac{\beta^{2}\left(1-\delta^{2}\right) R+\delta^{2}\left(1-\beta^{2}\right) S}{\left(1-\delta^{2}\right) R+\left(1-\beta^{2}\right) S}\right) \\
\geq\left(\frac{R \alpha \beta(1-\gamma \delta)+S \gamma \delta(1-\alpha \beta)}{R(1-\gamma \delta)+S(1-\alpha \beta)}\right)^{2} .
\end{gathered}
$$

Proof. We define

$$
\begin{aligned}
E= & \left(\frac{\alpha^{2}\left(1-\gamma^{2}\right) R+\gamma^{2}\left(1-\alpha^{2}\right) S}{\left(1-\gamma^{2}\right) R+\left(1-\alpha^{2}\right) S}\right)\left(\frac{\beta^{2}\left(1-\delta^{2}\right) R+\gamma^{2}\left(1-\alpha^{2}\right) S}{\left(1-\delta^{2}\right) R+\left(1-\alpha^{2}\right) S}\right) \\
& -\left(\frac{R \alpha \beta(1-\gamma \delta)+S \gamma \delta(1-\alpha \beta)}{R(1-\gamma \delta)+S(1-\alpha \beta)}\right)^{2} .
\end{aligned}
$$

Then

$$
E=\frac{R S(R+S)[(1-\gamma \delta) L \cdot R+(1-\alpha \beta) M \cdot S]}{\left[\left(1-\gamma^{2}\right) R+\left(1-\alpha^{2}\right) S\right]\left[\left(1-\delta^{2}\right) R+\left(1-\alpha^{2}\right) S\right][(1-\gamma \delta) R+(1-\alpha \beta) S]^{2}},
$$

where we have

$$
\begin{aligned}
L=\alpha^{2} & \beta^{2}(\alpha-\beta)^{2}+(\alpha-\beta)^{2} \gamma^{3} \delta^{3} \\
+ & \left\{(\alpha \delta)^{2}(1-\alpha \beta)\left(1+\alpha \beta-2 \beta^{2}\right)-(\alpha \delta)(\beta \gamma)\left(2-4 \alpha \beta+\beta \alpha^{3}+\alpha \beta^{3}\right)\right. \\
& \left.+(\beta \gamma)^{2}(1-\alpha \beta)\left(1+\alpha \beta-2 \alpha^{2}\right)\right\} \\
+ & \left\{\gamma^{2} \beta(1-\alpha \beta)\left(2 \alpha-\beta-\alpha \beta^{2}\right)-\gamma \delta\left(\alpha^{2}+\beta^{2}+2 \alpha^{3} \beta^{3}-4 \alpha^{2} \beta^{2}\right)\right. \\
& \left.+\delta^{2} \alpha(1-\alpha \beta)\left(2 \beta-\alpha-\alpha^{2} \beta\right)\right\} \gamma \delta,
\end{aligned}
$$


or, as a polynomial in $\gamma$ and $\delta$,

$$
\begin{aligned}
L= & \alpha^{2} \beta^{2}(\alpha-\beta)^{2}+\beta^{2}(1-\alpha \beta)\left(1+\alpha \beta-2 \alpha^{2}\right) \gamma^{2} \\
& +\alpha^{2}(1-\alpha \beta)\left(1+\alpha \beta-2 \beta^{2}\right) \delta^{2}-\alpha \beta\left(2+\alpha \beta^{3}-4 \alpha \beta+\beta \alpha^{3}\right) \gamma \delta \\
& +\beta(1-\alpha \beta)\left(2 \alpha-\beta-\alpha \beta^{2}\right) \gamma^{3} \delta-\left(\alpha^{2}+\beta^{2}+2 \alpha^{3} \beta^{3}-4 \alpha^{2} \beta^{2}\right) \gamma^{2} \delta^{2} \\
& +\alpha(1-\alpha \beta)\left(2 \beta-\alpha-\alpha^{2} \beta\right) \gamma \delta^{3}+(\alpha-\beta)^{2} \gamma^{3} \delta^{3}
\end{aligned}
$$

and where $M=M(\alpha, \beta, \gamma, \delta)=L(\gamma, \delta, \alpha, \beta)$. Thus it suffices to prove that for any $0<$ $\alpha, \beta, \gamma, \delta<1$ we have $L(\alpha, \beta, \gamma, \delta) \geq 0$. For this will also imply that $M(\alpha, \beta, \gamma, \delta) \geq 0$ for any such choice. This, in turn, will show that $E \geq 0$ for every choice of $R, S>0$ and $0<\alpha, \beta, \gamma, \delta<1$ and hence will prove Lemma 3. To check the nonnegativity of $L(\alpha, \beta, \gamma, \delta)$ we make the substitutions

$$
(\alpha, \beta, \gamma, \delta)=\left(\frac{x^{2}}{1+x^{2}}, \frac{y^{2}}{1+y^{2}}, \frac{z^{2}}{1+z^{2}}, \frac{w^{2}}{1+w^{2}}\right)
$$

and clear the denominators. This will give us a polynomial in $\mathbb{R}[x, y, z, w]$. In fact, this polynomial is

$$
\begin{aligned}
P(x, y, z, w)= & L\left(\frac{x^{2}}{1+x^{2}}, \frac{y^{2}}{1+y^{2}}, \frac{z^{2}}{1+z^{2}}, \frac{w^{2}}{1+w^{2}}\right) \\
& \times\left(1+x^{2}\right)^{4}\left(1+y^{2}\right)^{4}\left(1+z^{2}\right)^{3}\left(1+w^{2}\right)^{3} .
\end{aligned}
$$

As a consequence, it suffices to check that $P(x, y, z, w)$ is nonnegative for all real values of its indeterminates. We conclude the proof of Lemma 3 in the following section, after a brief detour explaining the sum of squares based methods we have used.

\section{Sums of Squares}

An obvious sufficient condition for nonnegativity is to represent $P(x, y, z, w)$ as a sum of squares of real polynomials. The connections between sums of squares and nonnegativity have been extensively studied since the end of the 19th century, when Hilbert showed that in the general case the two conditions are not equivalent. We refer the reader to the wonderful survey [11] by Reznick on the available results and history of Hilbert's 17th problem. In the work of Choi et al. [4] the algebraic structure of sums of squares decompositions is fully analyzed, and the important "Gram matrix" method is introduced. On the computational side, convex optimization approaches to this problem originate in the early work of Shor [14]. Recently, efficient techniques using semidefinite programming and exploiting problem structure have been developed in [9] and [5]. A brief description of the methods follows, referring the reader to the cited works, and the references therein, for the full algorithmic details.

We explain next the general idea of the Gram matrix method. Given a multivariate polynomial $F(\mathbf{x})$ for which we want to decide whether a sum of squares decomposition exists, we attempt to express it as a quadratic form in a new set of variables $\mathbf{u}$. A judicious 
choice of these new variables will depend on both the sparsity structure and symmetry properties of $F$ [4], [5]. For instance, for the simplest case of a generic dense polynomial of total degree $2 d$, the variables $\mathbf{u}$ will be all the monomials (in the variables $\mathbf{x}$ ) of degree less than or equal to $d$. Consequently, we try to represent $F(\mathbf{x})$ as

$$
F(\mathbf{x})=\mathbf{u}^{T} Q \mathbf{u},
$$

where $Q$ is a constant matrix. Since in general the variables $\mathbf{u}$ will not be algebraically independent, the matrix $Q$ in the representation (9) is not unique. In fact, there is an affine subspace of matrices $Q$ that satisfy the equality, as can be easily seen by expanding the right-hand side and equating term by term. If in the representation above the matrix $Q$ can be chosen to be positive semidefinite, then a factorization of the matrix $Q$ directly provides a sum of squares decomposition of $F(\mathbf{x})$. Conversely, if $F$ is a sum of squares, then such a $Q$ can always be constructed by expanding the terms in monomials. Therefore, the problem of checking if a polynomial can be decomposed as a sum of squares is equivalent to verifying whether a certain affine matrix subspace intersects the cone of positive definite matrices. This latter class of convex optimization problems is known as semidefinite programs (SDP) [16], and can be efficiently solved using a variety of numerical algorithms, mainly based on interior point methods.

Example 1. Consider the quartic form in two variables described below, and define $\mathbf{u}=\left[x^{2}, y^{2}, x y\right]^{T}$ :

$$
\begin{aligned}
F(x, y) & =2 x^{4}+2 x^{3} y-x^{2} y^{2}+5 y^{4} \\
& =\left[\begin{array}{c}
x^{2} \\
y^{2} \\
x y
\end{array}\right]^{T}\left[\begin{array}{lll}
q_{11} & q_{12} & q_{13} \\
q_{12} & q_{22} & q_{23} \\
q_{13} & q_{23} & q_{33}
\end{array}\right]\left[\begin{array}{c}
x^{2} \\
y^{2} \\
x y
\end{array}\right] \\
& =q_{11} x^{4}+q_{22} y^{4}+\left(q_{33}+2 q_{12}\right) x^{2} y^{2}+2 q_{13} x^{3} y+2 q_{23} x y^{3} .
\end{aligned}
$$

Therefore, in order to have an identity, the following linear equalities should hold:

$$
q_{11}=2, \quad q_{22}=5, \quad q_{33}+2 q_{12}=-1, \quad 2 q_{13}=2, \quad 2 q_{23}=0 .
$$

A positive semidefinite $Q$ that satisfies the linear equalities can then be found using semidefinite programming. A particular solution is given by

$$
Q=\left[\begin{array}{rrr}
2 & -3 & 1 \\
-3 & 5 & 0 \\
1 & 0 & 5
\end{array}\right]=L^{T} L, \quad L=\frac{1}{\sqrt{2}}\left[\begin{array}{rrr}
2 & -3 & 1 \\
0 & 1 & 3
\end{array}\right]
$$

and therefore we have the sum of squares decomposition:

$$
F(x, y)=\frac{1}{2}\left(2 x^{2}-3 y^{2}+x y\right)^{2}+\frac{1}{2}\left(y^{2}+3 x y\right)^{2} .
$$

Back to our concrete problem, the polynomial $P$ in (8) has four variables, 123 nonzero monomial terms and total degree 20 . Notice that a polynomial of that degree and number of variables generically has $\left(\begin{array}{c}20+4 \\ 4\end{array}\right)=10,626$ monomials, so $P$ is quite sparse. In 
particular, it has a bipartite structure, with degree 12 in $x, y$, and degree 8 in $z, w$. Furthermore, $P$ has very appealing symmetry properties: some inherited from $L$, and some as a result of the substitution (7). Concretely, it is easy to see that $P$ is invariant under the transformations

$$
\begin{aligned}
(x, y, z, w) & \rightarrow(y, x, w, z) \\
& \rightarrow( \pm x, \pm y, \pm z, \pm w) .
\end{aligned}
$$

The first property is a clear consequence of the symmetry of our original geometric inequality with respect to the interchange of the two triangles. The second one is a side effect of our choice for modeling the nonnegativity constraints. The transformations given above generate a symmetry group $G$ with 32 elements and 14 irreducible representations: eight one-dimensional and six two-dimensional $\left(8 \cdot 1^{2}+6 \cdot 2^{2}=32\right)$. As explained extensively by Gatermann and Parrilo in [5], these symmetries can be exploited very successfully in reducing the computational requirements.

To do this, the approach in [5] relies on a crucial property of convex optimization problems invariant under a group action, namely the fact that the optimal solution can always be restricted by a subspace fixed by the group action. Using Schur's lemma of representation theory, it is shown there that by using an appropriate symmetry-adapted coordinate transformation, the original SDP can be decomposed into a collection of smaller coupled problems, of cardinality equal to the number of irreducible representations of the group. This reduces both the size and the number of variables in the problem, and as a consequence notably enhances both the accuracy and conditioning of the solution.

Attempting to establish the nonnegativity of $P$ directly without taking into account both the sparsity and symmetries can be a difficult task for current SDP solvers, both in terms of memory requirements and accuracy. A naive approach, using only degree information but no structure whatsoever, would require solving an SDP of dimension $1001 \times 1001$ and 10,626 constraints. By exploiting only the sparsity of $P$, but not its symmetry, the problem is reduced to dimension $137 \times 137$ and 1328 constraints. Adding the simplifications resulting from the symmetries, the problem is further simplified to a much more manageable one with 14 coupled SDPs (one per each irreducible representation) of dimensions ranging between $2 \times 2$ and $11 \times 11$ (see Table 1). For instance, for the trivial irreducible representation (number 1 in the table), the corresponding new variables $\mathbf{u}$ are invariant under the group action, and given by

$$
\begin{gathered}
y^{2} z^{2}+x^{2} w^{2}, \\
x^{2} z^{2} w^{2}+y^{2} z^{2} w^{2}, \quad y^{4} z^{2}+x^{4} w^{2}, \quad x^{2} y^{2} z^{2}+x^{2} y^{2} w^{2}, \quad x^{4} y^{2}+x^{2} y^{4}, \\
x^{2} y^{2} z^{2} w^{2}, \quad x^{4} z^{2} w^{2}+y^{4} z^{2} w^{2}, \quad x^{2} y^{4} z^{2}+x^{4} y^{2} w^{2}, \quad x^{4} y^{2} z^{2}+x^{2} y^{4} w^{2} .
\end{gathered}
$$

Notice in Table 1 that the combined total, taking into account multiplicities, is equal to 137 , the dimension of the sparse version of the problem.

The resulting system of matrix inequalities can be solved with standard SDP solvers, such as SeDuMi [15]. The output provides a decomposition of $P$ as a sum of squares of polynomials, with coefficients given by floating point numbers. In this particular case, the computed values immediately suggest the existence of a solution, presented below, with polynomials having integer coefficients. The solution can be verified in a completely independent fashion, providing a mathematically correct certificate of the nonnegativity of the polynomial $P$. 
Table 1. Irreducible representations of $G$ and the corresponding SDP dimensions.

\begin{tabular}{lrrrrrrrrrrrrrr}
\hline & \multicolumn{11}{c}{ Irreducible representation number } \\
\cline { 2 - 14 } & 1 & 2 & 3 & 4 & 5 & 6 & 7 & 8 & 9 & 10 & 11 & 12 & 13 & 14 \\
\hline Multiplicity & 1 & 1 & 1 & 1 & 1 & 1 & 1 & 1 & 2 & 2 & 2 & 2 & 2 & 2 \\
Dim. SDP & 9 & 6 & 6 & 4 & 8 & 5 & 3 & 2 & 11 & 7 & 8 & 7 & 8 & 6 \\
\hline
\end{tabular}

Proof of Lemma 3 (continued). Consider the following three polynomials:

$$
\begin{gathered}
A(x, y, z, w)=-y^{2} z^{2}-y^{4} z^{2}+x^{2} w^{2}+2 x^{2} y^{2} w^{2}-2 x^{2} y^{2} z^{2}-x^{2} y^{4} \\
-2 x^{2} y^{4} z^{2}+x^{4} w^{2}+x^{4} y^{2}+2 x^{4} y^{2} w^{2} \\
B(x, y, z, w)=\left(1+x^{2}+y^{2}\right)\left(-x^{2} w^{2}-x^{2} z^{2} w^{2}-x^{2} y^{2} w^{2}+x^{2} y^{2} z^{2}\right. \\
\left.+y^{2} z^{2}+y^{2} z^{2} w^{2}\right)
\end{gathered}
$$

and

$$
\begin{gathered}
C(x, y, z, w)=(x-y)(x+y)\left(-x^{2} z^{2} w^{2}+x^{2} y^{2}+x^{2} y^{2} w^{2}+x^{2} y^{2} z^{2}\right. \\
\left.-z^{2} w^{2}-y^{2} z^{2} w^{2}\right) .
\end{gathered}
$$

Then we have the following identity:

$$
P(x, y, z, w)=A(x, y, z, w)^{2}\left(z^{2}+w^{2}+2 z^{2} w^{2}\right)+B(x, y, z, w)^{2}+C(x, y, z, w)^{2} .
$$

Thus $P(x, y, z, w)$ is a sum of five squares of real polynomials and the proof of Lemma 3 is completed.

Rewriting the obtained sum of squares decomposition in terms of the original variables, the following representation of $L$ can be obtained:

$$
\begin{aligned}
L & =L_{1}+L_{2}+L_{3}, \\
L_{1} & =(\gamma+\delta)\left(-\alpha^{2} \beta+\alpha \beta^{2}-\alpha \delta+\beta \gamma-\beta \gamma \delta+\alpha \delta \gamma-\alpha \beta^{2} \gamma+\alpha^{2} \beta \delta\right)^{2}, \\
L_{2} & =(1-\gamma)(1-\delta)(\alpha \beta-1)^{2}(\alpha \delta-\beta \gamma)^{2}, \\
L_{3} & =(1-\gamma)(1-\delta)(\alpha-\beta)^{2}(\alpha \beta-\gamma \delta)^{2} .
\end{aligned}
$$

From this, stronger conclusions on the sign of $L$ can be immediately derived: not only is it nonnegative on the open unit hypercube $(0,1)^{4}$ as needed for Lemma 3 , but the same property holds on the much larger region $\mathbb{R} \times \mathbb{R} \times\{\gamma+\delta \geq 0,(1-\gamma)(1-\delta) \geq 0\}$.

\section{Acknowledgments}

We warmly acknowledge the help of Bruce Reznick, who made possible this collaboration by introducing the authors to each other's work. We also thank the reviewers for their useful comments and suggestions that helped improve the paper. 


\section{References}

1. Symposium on the Occasion of the Proof (1985: Purdue University), The Bieberbach conjecture: Proceedings of the Symposium on the Occasion of the Proof, American Mathematical Society, Providence, RI, 1986.

2. C. Bessaga, On the converse of the Banach "Fixed Point Principle", Colloq. Math., 7 (1969), 41-43.

3. A. Berman and R. J. Plemmons, Nonnegative Matrices in the Mathematical Sciences, Academic Press, New York, 1979.

4. M. D. Choi, T. Y. Lam, and B. Reznick. Sums of squares of real polynomials. Proc. Sympos. Pure Math., 58(2) (1995), 103-126.

5. K. Gatermann and P. A. Parrilo. Symmetry groups, semidefinite programs, and sums of squares. Submitted, available from arXiv: math. AC/0211450, 2002.

6. A. S. Householder, The Theory of Matrices in Numerical Analysis, Dover, New York, 1964.

7. A. Marden and B. Rodin, On Thurston's formulation and proof of Andreev's theorem, in Computational Methods and Function Theory, LNM 1435, Springer-Verlag, New York, 1989, pp. 103-115.

8. J. M. Ortega and W. C. Rheinboldt, Iterative Solution of Nonlinear Equations in Several Variables, Academic Press, New York, 1970.

9. P. A. Parrilo, Semidefinite programming relaxations for semialgebraic problems. Math. Programming, to appear, doi:10.1007/s10107-003-0387-5. Available from http://control. ee.ethz.ch/ parrilo, 2001.

10. R. Peretz, Iterations of concave maps, the Perron-Frobenius theory and applications to circle packings, Electron. J. Linear Algebra, 9, (2002), 197-254.

11. B. Reznick, Some concrete aspects of Hilbert's 17th problem, in Contemporary Mathematics, volume 253, pages 251-272, American Mathematical Society, Providence, RI, 2000.

12. B. Rodin and D. Sullivan, The convergence of circle packings to the Riemann mapping, J. Differential Geom., 26 (1987), 349-360.

13. E. Seneta, Non-negative Matrices: An Introduction to Theory and Applications, Wiley, New York, 1973.

14. N. Z. Shor, Class of global minimum bounds of polynomial functions. Cybernetics, 23(6) (1987), 731-734. (Russian orig.: Kibernetika, No. 6, (1987), 9-11).

15. J.F. Sturm, Using SeDuMi 1.02, a MATLAB toolbox for optimization over symmetric cones, $O p$ tim. Methods Software, 11-12 (1999), 625-653. Version 1.05 is available at http://fewcal. kub.nl/sturm/software/sedumi.html.

16. H. Wolkowicz, R. Saigal, and L. Vandenberghe, editors, Handbook of Semidefinite Programming, Kluwer, Dordrecht, 2000.

Received June 3, 2002, and in revised form April 15, 2003. Online publication February 9, 2004. 